\title{
A NOVEl DATA DiCTIONARY LEARNING FOR LEAF RECOGNITION
}

\author{
Shaimaa Ibrahem ${ }^{1}$, Yasser M. Abd El-Latif ${ }^{2}$ and Naglaa M. Reda ${ }^{2}$ \\ ${ }^{1}$ Higher Institute for Computer Sciences and Information System, Egypt. \\ ${ }^{2}$ Faculty of Sciences, Ain Shams University, Egypt. \\ ORCID: 0000-0003-0701-087X
}

\begin{abstract}
Automatic leaf recognition via image processing has been greatly important for a number of professionals, such as botanical taxonomic, environmental protectors, and foresters. Learn an over-complete leaf dictionary is an essential step for leaf image recognition. Big leaf images dimensions and training images number is facing of fast and complete data leaves dictionary. In this work an efficient approach applies to construct over-complete data leaves dictionary to set of big images diminutions based on sparse representation. In the proposed method a new cropped-contour method has used to crop the training image. The experiments are testing using correlation between the sparse representation and data dictionary and with focus on the computing time.
\end{abstract}

\section{KEYWORDS}

Leaf image recognition, Dictionary learning, Sparse representation, Online Dictionary Learning

\section{INTRODUCTION}

Recognition has become an important technique in various fields for the past few years. recognition algorithms are popular for many kinds of applications such as face recognition, action recognition and leaf recognition [1]. This research has focused on leaf recognition based on the analysis of leaf images. The challenging task in the Leaf recognition from leaf images is to find discriminant features that can be appropriate for distinguishing different leaves classes. In order to classify the leaf different characteristics have been evaluated such as color, shape, texture, morphology and venation structure as comparative tool, and some leaf datasets such as Swedish leaf dataset, Flavia dataset, and ICL dataset are standard benchmark.

Most of the researchers focus on the leaf shape features, because the overall shape structure of a leaf may be preserved even though the leaf sample being damaged by age or insects but also the leaf shape is approximate same in large set of leaves.

A shape-based approach for leaf classification presented in many researches [1-5]. Jou-ken [1] presented a planet identification system based on shape-based using feature extracted from the leaf images by scale invariant feature transform (SIFT) method. Yang et al. [2] presented a new approach for plant leaf recognition using contour-based shape descriptor called multi-scale triangular centroid distance (MTCD) and dynamic programming (DP). This descriptor extracts The MTCD features from each contour point to provide a compact, multi-scale shape descriptor. The DP program finds the best alignment between corresponding points of the shapes. Also Wu, S.G. [3] developed plant identification system based on neural network using a feed forward neural network (PNN) probabilistic neural network. Same plant leaf recognition presented in [4] by using move median center method (MMC)hyper sphere classifier. 
Mzoughi et al. [6] proposed a leaf recognition system depend on split the entire leaf image to three parts, top, middle and base, and then based on contour and texture for each part of the leaf search for candidate species through the fusion of descriptors. The author integrated the proposed structuring process using a retrieval decision with a k-NN classifier and pointed out the reduction of the leaf variety complexity within and across species as the main advantage, since it enables the definition of pertinent features for each subspace (part), depending on its discriminatory properties.

Aakif and Khan [7] proposed a novel leaf recognition algorithm using depend on shape-based with different features such as morphological characters, Fourier descriptors and a newly designed Shape-Defining Feature (SDF). The algorithm showed its effectiveness in baseline dataset like Flavia.

Recently, many researchers presented a combination characteristic for plant leaf recognition $[8$, 9]. Kadir [8] present plant identification depend on set of characteristics as color, texture and shape extracted from the leaves images. Olsen et al. [9] proposed a leaf classification situ using rotation and a scale invariant histograms of oriented gradients (HOG) feature set to represent regions of texture within leaf images. Tang et al. [10] introduced a novel texture extraction method, based on the combination of Gray Level Co-Occurrence Matrix (GLCM) and local binary pattern (LBP), to classify green tea leaves. G.L. Grinblat. [11] Present a new plant identification using vein morphological patterns. This algorithm first extracted the vein patterns using Hit or Miss Transform (UHMT), and then trained a convolutional neural network (CNN) to identify them using a set of central patch of leaf images. In addition, a considerable amount of research has used combinations of features to represent leaves.

An interesting approach to deal with the various leaf feature and huge number of leaves is the online dictionary learning (ODL) as in our proposed approach. Online Sparse Dictionary Learning algorithm (OSDL) is a famous algorithm used to build dictionary learning. Most recent dictionary learning is performing by algorithms such as the online dictionary learning (ODL) [12] which is used in Learning-Based Leaf Image Recognition Frameworks [1]. Another algorithm performing data dictionary is K-singular value decomposition (K-SVD) [13]. These algorithms achieved efficiently with images haves small pixel dimensions and usually take long time in computing. This paper will concentrate on (ODL) algorithm dealing with large pixel dimension images.In this work we present Leaf recognition system that find discriminant features that can be appropriate for distinguishing between different leaves classes depend on leaf's sparse representation. We reach accuracy $96 \%$ in all of our experiment.

The rest of this paper is proceeds as follows. The methodology, pre-processing operations, feature extraction, build the online data dictionary are explained in Section 2, whie the experimental results are presented and evaluated in Section 3. Section 4 concludes the paper with a summary of the proposed work.

\section{MeTHOdOLOGY}

The proposed system is divided into two stages training stage and testing stage. The training stage start with pre-processing operations for the training image set. Then the sparse representation is computed for all training image set and used to build the data dictionary learning (DL) for all classes in dataset separately. In testing stage the sparse representation for the testing image in testing set is computed and then comparing with the data dictionary depend on correlation method; the best matching refer to the class that testing image is belong to. 
Signal \& Image Processing: An International Journal (SIPIJ) Vol.10, No.3, June 2019

The two stages in our proposed algorithm are described as follow:

\subsection{Training stage:}

Step1: pre-processing operations (extract the leaf from the training image using the contour).

Step2: patching the training leaf image set for all leaves in the different classes separately (feature extraction).

Step3: compute the sparse code for patches (sparse representation).

Step4: build the (ODL) to different leaf classes.

\subsection{Testing stage:}

Step1: compute the sparse code for the testing image set.

Step2: compute the correlation for the testing image and all (ODLs).

Step3: compare the correlation results. The greatest correlation refer to the class that test image is belong to (classification process).

We describe in the following paragraphs the proposed method in details:

\subsubsection{Pre-processing:}

In all leaves dataset the leaves were scanned on a white sheet (background).We consider here a set of training images from ( 1 to $n$ ) .To classifies the leaf we cropped it from the background (discard the w blocks) depend on contour edges. The colored images is converted to grayscale image by applying rgb2gray then converted to a binary image using im $2 \mathrm{bw}$ function. Then theleaf contour is extracted.

\subsubsection{Feature Extraction:}

In this step, each image resulting from the previous pre-processing method is submitted to the extraction process in order to obtain different descriptors from each image. The descriptors extracted here are the image patches.

In the proposed system there is many ways to patches the leaf image; if we want a certain number of patches we can add boundary column to fit the number of patches required; also we can delete or ignore columns to fit the required patches number. Otherwise if we don't determine the number of patches as we do in this paper we determine the coordinate of one patch and get patches from the input image as available.

\subsubsection{Sparse representation:}

In this step, sparse representation is computed for the training image set. Sparse representation is a representation method which aims to finding a sparse code for the input data in the form of a linear combination of basic elements. These elements are called atoms. 
Sparse representation of an image over complete dictionary is achieved by optimizing a sparse dictionary which is a matrix with sparse coding for $\mathrm{K}$ patches or feature vectors extracted from training set images $\mathrm{C}$ with length $\mathrm{N}$. Sparse coding, modeling as sparse linear combinations by basic dictionary (atoms) and sparse coefficient as show in figure 1.

A sparse dictionary [15] form as a sparse structure $D=B * A$ Where, $\mathrm{B}$ is a fixed base dictionary and $A$ is a sparse matrix. The matrix $D$ is a sparse dictionary matrix of size $\mathrm{N} \times \mathrm{K}$, containing the sparse representations of the dictionary atoms over B.The base dictionary B typically has a fast algorithmic implementation \{Specifically, faster than explicit matrix multiplication\} this makes the sparse dictionary very efficient to apply.

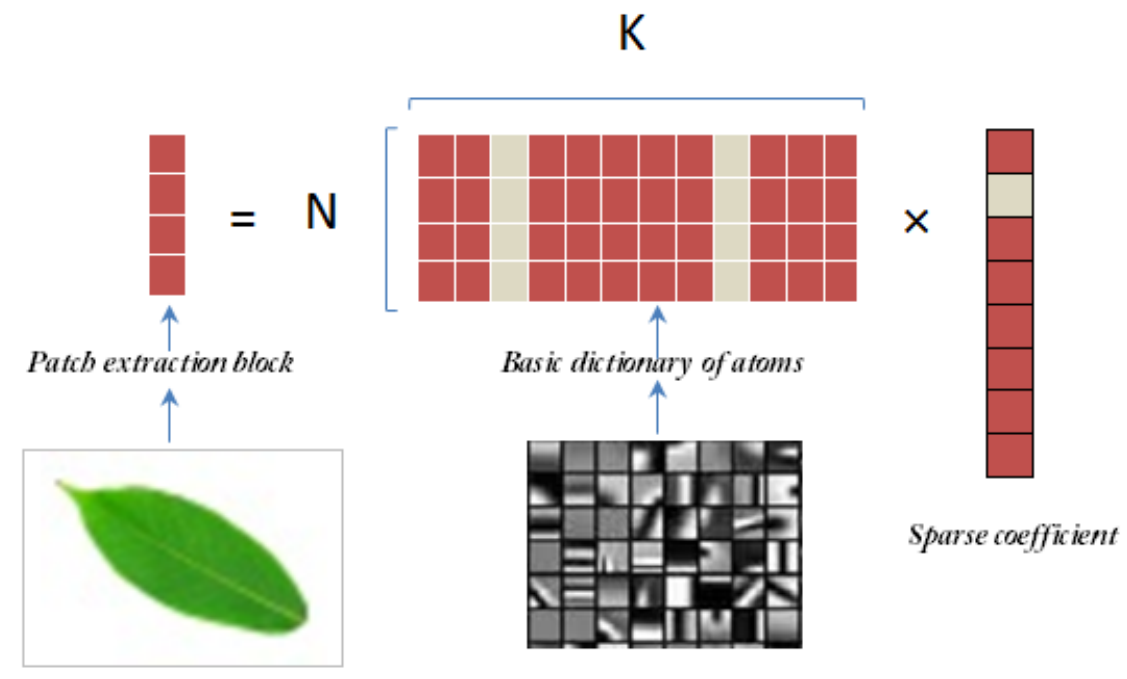

Figure 1: An example of illustrating sparse representation of extracting patches from training images set

\subsubsection{Online Dictionary Learning based on Sparse Representation}

Data Dictionary learning is a dictionary of atoms that have the good sparse coefficient. It should be small with good data representation. Dictionary learning $D$ for sparse representation here is a collection of atoms (training patches) of length $N \times K$ where $N$ is atom vector length and $k$ is the number of atoms. The dictionary learning problem is finding a dictionary such that the approximation of the training set is good as possible given a spars coefficient $w$ and $x$ is a set of patch extraction; this problem can be written as

$$
x=D w
$$

There is infinity many possible solutions to the system $x=D w$. Among this infinitely large set of solutions, the sparse representation is preferred with smallest $\|x\|_{o}-$ norm. Thus, the task of computing D for a signal can be formally evaluated by

$$
\underset{w}{\min }\|x\|_{o} \text { Subject to } x=D w
$$

The exact equation in the constraint above is replaced by the alternative requirement $\| x-$ $D w \|_{2}$ to allow additive noise and model deviations. 
After collecting a set of vectors of $y_{j}$, the training patches exemplars, $j=1,2, \ldots, P$, with respect to $\mathrm{D}$ Where $\mathrm{w}_{\mathrm{j}}$ denotes the sparse coefficient and $\lambda$ is a regularization parameter to learn a dictionary D sparsifyingy, the following optimization equation solved [8]:

$$
\min _{D, w_{j}} \Sigma_{j}^{p} \sum_{j=1}\left(\frac{1}{2}\left\|y_{j}-D w_{j}\right\|_{2}^{2}+\lambda\left\|w_{j}\right\| 1\right)
$$

\subsubsection{Sparse representation for the testing image set:}

In this step, sparse representation for the testing image set is computed for every image in test set separately.

\subsubsection{Correlation:}

Correlation is a statistical technique that is used to measure and describe the strength and direction of the relationship between two variables. It is obtained by dividing the covariance of the two variables by the product of their standard deviations. Karl Pearson developed the coefficient from a similar but slightly different idea by Francis Galton[16]. After we extract the sparse representations as $\mathrm{w}$ for a test image then we get the correlation between $\mathrm{w}$ and the data dictionary $\mathrm{D}$ with expected values $\mu \mathrm{w}$ and $\mu \mathrm{D}$ and standard deviations $\sigma \mathrm{W}$ and $\sigma \mathrm{D}$ is defined as:

$$
\operatorname{Corr}(\mathrm{W}, \mathrm{D})=\frac{\left(\mathrm{w}-\mu_{w}\right)\left(\mathrm{D}-\mu_{D}\right)}{\sigma w \sigma D}
$$

corr is a widely used alternative notation for the correlation coefficient.

\subsubsection{Classification process:}

In this step, we classification the testing image set by correlation results that obtained according to correlation the sparse coefficients of the test image set and class data dictionary. The class associated with the maximum correlation with the input image will be decided to be the class that the input image belongs to.

\section{EXPERIMENT AND RESULT ANALYSIS}

\subsection{Experimental environment:}

The system of recognition used was to use image of the leaves in the data set "Flavia leaf image dataset"[16] a popular leaf image dataset. It dataset consists of 32 classes of leaf images ( $c=32$ ) where each class contains 40-60 images. Table 1 showing some of dataset leaves.

Our experiments were implemented in matlab R2014a (64 bits version) on a personal computer equipped with Intel ${ }^{\circledR}$ core ${ }^{\mathrm{TM}} \mathrm{i} 5-2410 \mathrm{M}$ processor. Moreover function and tools using within matlab all the experiment use ompboxl ,ompbox10 multi-threaded $\mathrm{C}$ coded loops and mtimesx_20110223 in sparse code level. MATLAB does not always implement the most efficient algorithms for memory access, and MATLAB does not always take full advantage of symmetric and conjugate cases. MTIMESX $X^{3}$ attempts to do both of these to the fullest extent possible, and in some cases can outperform MATLAB by $3 \mathrm{x}-4 \mathrm{x}$ for speed faster. 
Signal \& Image Processing: An International Journal (SIPIJ) Vol.10, No.3, June 2019

Table 1: Examples of leaf Data set images

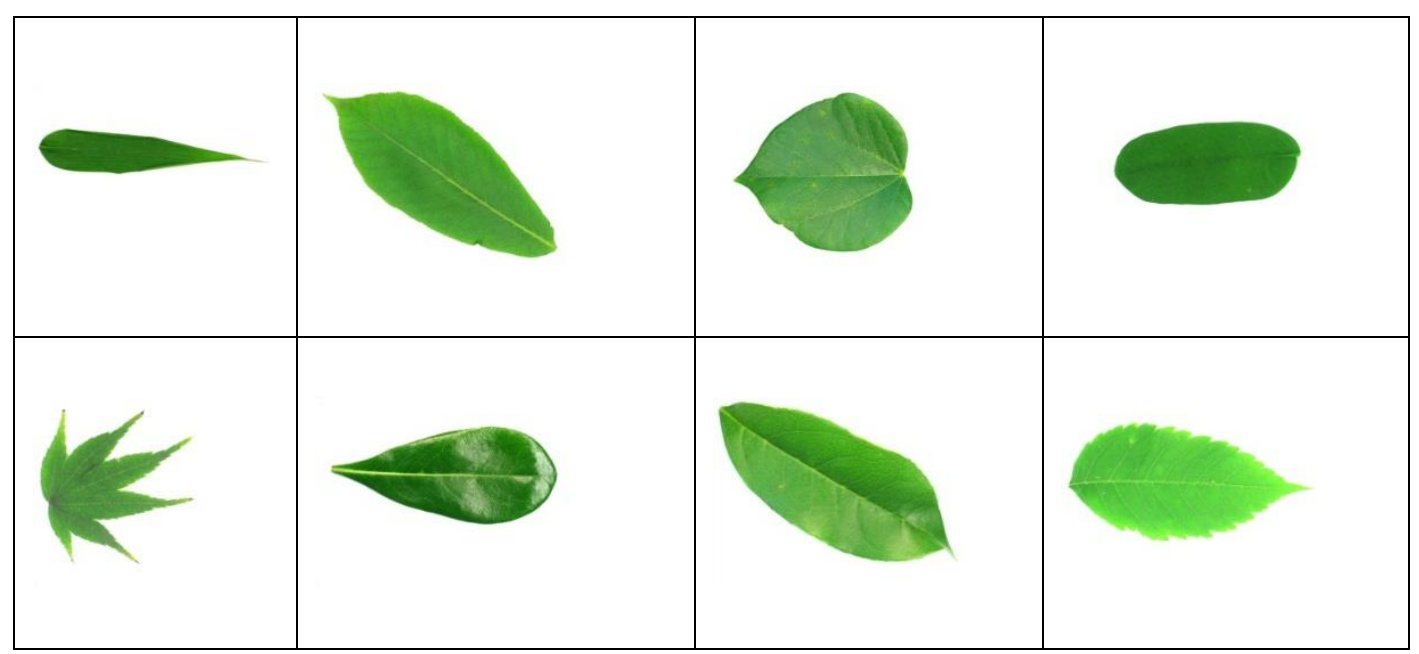

\subsection{Experimental method}

Experiments is to formalization sparse representation and data dictionary learning to all images in a $C$ class $\mathrm{i}=1,2, . ., \mathrm{c}$ for the extracting patches $P_{i}$ with different size $(16 \times 16,32 \times 32,60 \times 60)$.In this experiment we study the relation between patch size and recognition rate and study the execution time. Testing was done on 32 different leaf types every type consists about 60 leaf images. In particular, we noted $100 \%$ accuracy for different types using small data dictionary size. Few leaf images are removed from experiment because the extracted features are not enough. The result of all experiment is discus next.

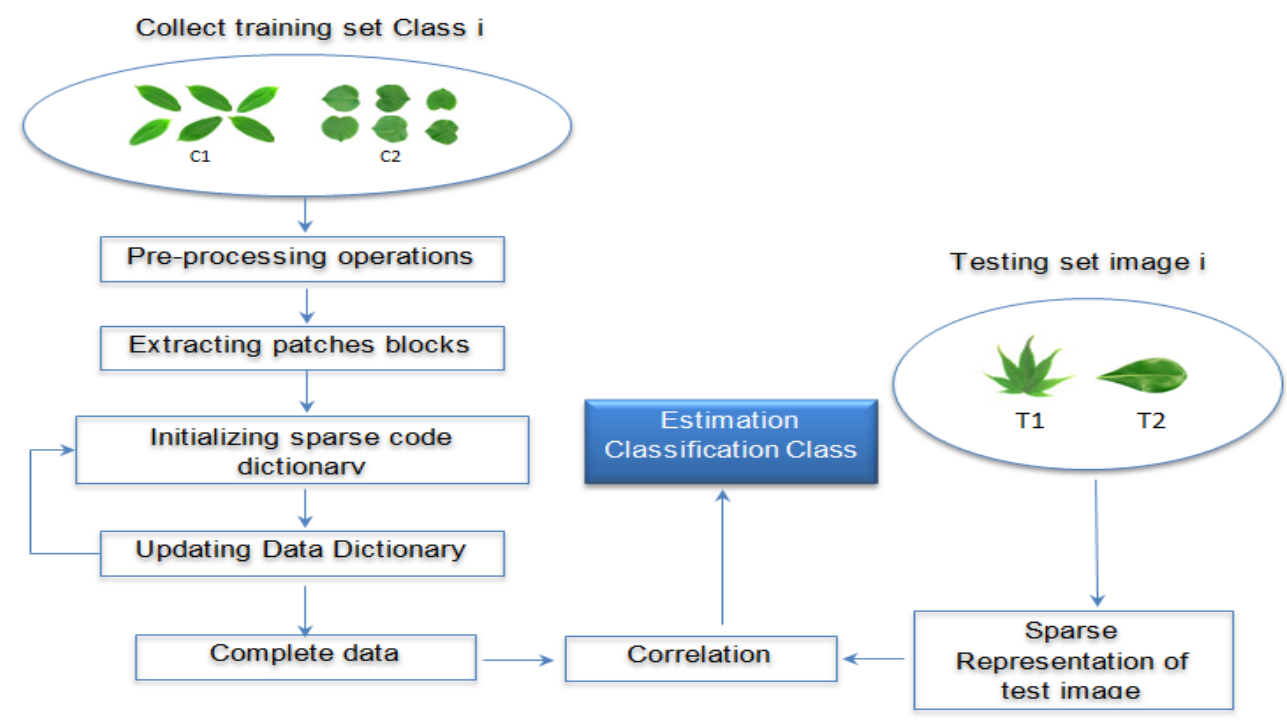

Figure 2: The proposed leaf image recognition steps

\subsection{Experimental Results}




\subsubsection{Recognition rate:}

Firstly experiments, Recognition rate has achieved in our proposed image recognition system. The following paragraphs explain our experiments.

To study the efficiency whether the increase in the number of images in learning is useful or not, we performed the first experiment using a few images (10 images of size $1200 \times 1600)$ to build the data dictionary and then doubled the number of images. In this experiment we extracted the leaf boundary and used different patches sizes $(16 \times 16,32 \times 32)$ to extract the patches. The goal is to access the full data dictionary for all available images with large image dimensions and using the appropriate patches while maintaining the number of images required in the education without computational load increase. Table 2 present Examples to boundary leaf images.

Table 2: Examples of leaf images in cropped stage

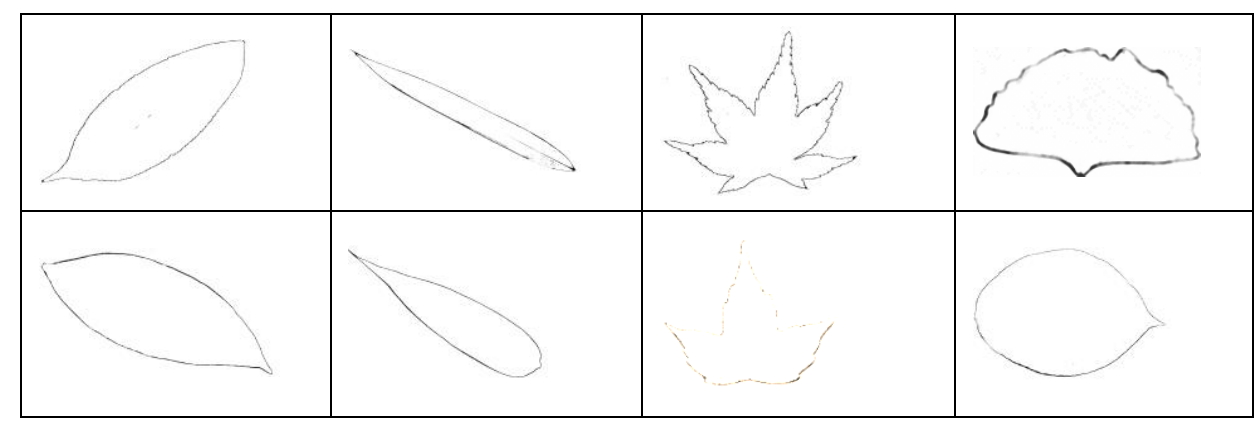

The patches function run in two ways, either extracting all available patches in the cropped leaf image, or in the case of experiments that the image size is large and the patch size is small, for example $16 \times 16$, the extracted patches may be greater than million samples and it made load over when compute the sparse code, so that a certain number of patches must be determine to extract. In the experiments with patch size is $32 \times 32$, the patch number increase between 10000 up to 1 million patches depend on the number of training images.

The tests were performed using three levels. In first level the testing set contains leaf images used in learning stage. In the second level the testing set contains leaf images of the same class but not used in learning stage. The third class contains similar leaf images from different classes.

The result of the previous experiment obtained by Appling the proposed algorithm to two data dictionary, dictionary 1 which contains a number of images to training and dictionary 2 contains the double of dictionary 1 .

The results showed that the training images number is effective in recognition rate. where increase the training images the recognition rate increases until the data dictionary is completed with features of the leaf then the addition in training image occurs overload in computation and overload in comparison time where it decrease the recognition rate.

Also our proposed algorithm work in the same dataset presented in $[1,3,4,5,8]$. Table 3 lists the recognition rates obtained by bag-of-words (BoW) presented in [1], sparse coding (SC) presented in [1], probabilistic neural network-Based (PNN) presented in [3], move median centers method (MMC) presented in [4], Proposed- method, respectively. In [1] randomly 30 image is selected in the learning stage for building OSDL using sparse representation for feature extracted using SIFT 
with length $\mathrm{n}=128$ for each descriptor, which is small dimension and tack long time in computation, while the PNN approach proposed in [9] used 1,800 training images for neural network training also take long time.

In Table 3, the recognition rates obtained by $\mathrm{Wu}$ et al. [3] and Du et al. [4] were reported in [3], while Jou-Ken[1] reported BoW And SC were their respective beter than [3] and [4]. Compared with the BoW-based approach, the main advantage of SC-based approach is that it is not required to re-train classifiers with newly leaf image class added, while in the BoW-based approach, both the codebook and the SVM classifier are required to be re-trained.

It can be observed from Table 3 that the proposed method are comparable with the six existing approaches used for comparisons (BoW and SC [1], PNN [9], MMC [11], HPNN [13], and CShape [14]).

Table 3 : present the recognition improve results

\begin{tabular}{ll}
\hline Method & Rate \\
\hline BoW [1] & 94.38 \\
SC [1] & 95.47 \\
PNN [3] & 90.31 \\
MMC [4] & 91 \\
CShape [5] & 94.62 \\
HPNN [8] & 93.75 \\
Proposed method & 96 \\
\hline
\end{tabular}

\subsubsection{Accelerating time:}

Secondly experiments, focus on achieve time reducing in dealing with large size images and large patch size in image recognition. The following paragraphs explain our experiments.

In the previous experiment we were dealing with large size images and patch size $16 \times 16$ and $32 \times 32$. Here we increase the patch size to $60 \times 60$ and replay building data dictionary and replay the correlation for the same training and testing images. Table 4 present the result to this experiment compared with the previous experiment results.

Table 4 : present a comparison of the experimental results using various patch size

\begin{tabular}{lllll}
\hline Patch size & $\begin{array}{l}\text { OSDL size for 40 training } \\
\text { images }\end{array}$ & $\begin{array}{l}\text { Building } \\
\text { time }\end{array}$ & $\begin{array}{l}\text { File size } \\
\text { (.mat) }\end{array}$ & $\begin{array}{l}\text { Recognition } \\
\text { rate }\end{array}$ \\
\hline $\mathbf{1 6 x 1 6}$ & $256 \times 100000$ & $18000 \mathrm{~s}$ & $600 \mathrm{~Kb}$ & 96 \\
$\mathbf{3 2 \times 3 2}$ & $1024 \times 100000$ & $54000 \mathrm{~s}$ & $2.22 \mathrm{Mb}$ & 96.5 \\
$\mathbf{6 0 x 6 0}$ & $3600 \times 100000$ & $208800 \mathrm{~s}$ & $4.88 \mathrm{Mb}$ & 94 \\
\hline
\end{tabular}

In this big size of patch construct the sparse dictionary is slow and because of the amount of training data is limited the dictionary is weak and perhaps insufficient to train a full dictionary. Also we include the result presented in [18] "Trainlets: Dictionary Learning in High Dimensions" that paper proposed a modification on the Wavelet transform by constructing two-dimensional separable cropped Wavelets. The data dictionary employing using SGD ideas in the dictionary learning task. It used to perform Image Restoration Demonstration. The experiments were perform on patches of size $64 \times 64$ using ODL to building the data dictionary and the run time is 120 hour to show the ODL schema with higher dimensional signal. In our experiment the run time is 58 hour for $60 \times 60$ patch size.

\section{CONCLUSIONS AND FUTURE WORK}


In this paper we proposed a new method to recognition leaves using the OSDL method. We evaluated the performance of our proposed method according to the recognition rate and execution time. The recognition rate achieved was $96 \%$, and accelerating time achieved was $62 \%$. There are several facts are presented based on experiments include:

1) Increasing in training image achieve increase in recognition rate until reaching the full data dictionary. In then increasing in training image will occur overload in testing stage.

2) Whine increasing the patches size the non-zero sparse representation is increasing also increasing the computational time as show in figure 2 .

3) Increasing in patches size produce limit number of patches so insufficient to train a full dictionary and decrease the recognition rate as show in figure 3.

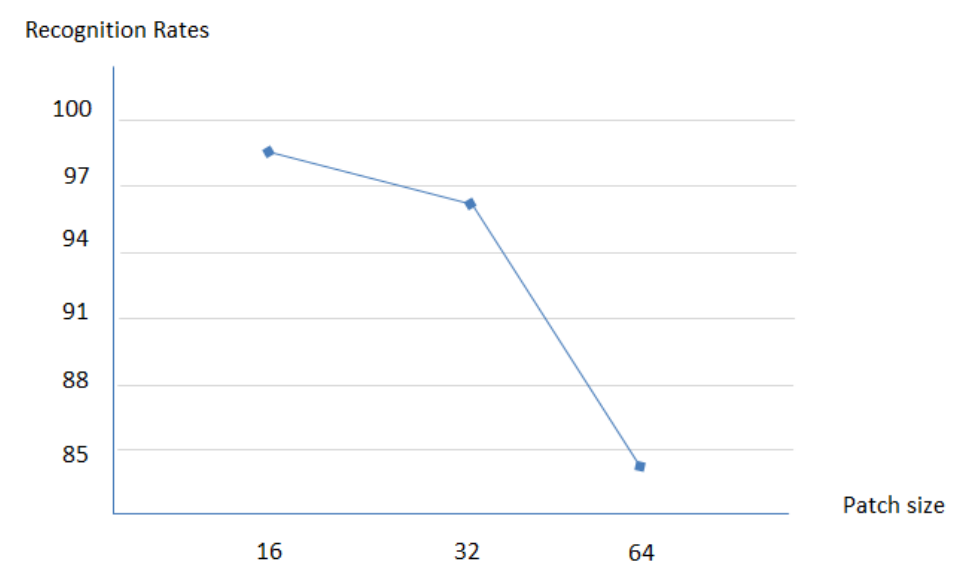

Figure 3: performance comparison between patch size depend on recognition rate

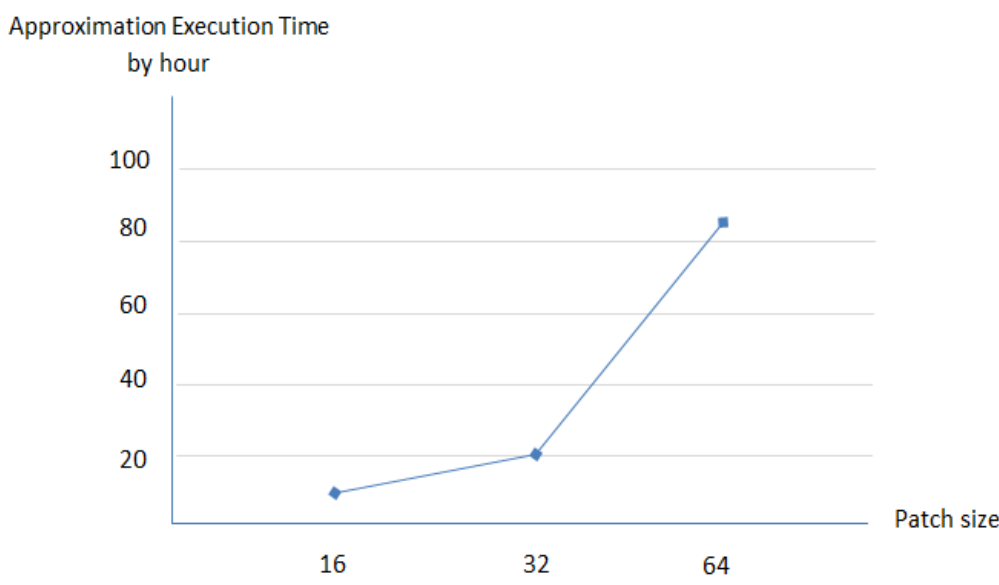

Figure 4: performance comparison patch size depend on execution time

This paper shows that dictionary learning can be up-scaled to tackle a new level of large signal dimensions. In future research we will attempt to achieve better result by improve in:

1) contour-cropped method to extract all features related to main object from all training images.

2) To build a full data dictionary for more training images we need to improve the computing method as using parallel computing that aim to accelerate the execution time.

\section{REFERENCES}


[1] Jou-Ken Hsiao, Li-Wei Kang, "Learning-Based Leaf Image Recognition Frameworks", Springer International Publishing Switzerland 2015.

[2] C. Yang, H. Wei, and Q. Yu, "Multiscale Triangular Centroid Distance for Shape-Based Plant Leaf Recognition," in European Conf. on Artificial Intelligence, 2016, pp. 269-276.

[3] Wu, S.G., Bao, F.S., Xu, E.Y., Wang, Y.-X., Chang, Y.-F., Xiang, Q.-L.” A leaf recognition algorithm for plant classification using probabilistic neural network.", In: Proceedings of IEEE International Symposium on Signal Processing and Information Technology, pp. 11-16,Giza, Egypt Dec 2007.

[4] Du, J.-X., Wang, X.-F., Zhang, G.-J. ” Leaf shape based plant species recognition. “ Appl. Math.Comput. 185(2), 883-893 (2007).

[5] Sari, C., Akgul, C.B., Sankur, B., " Combination of gross shape features, fourier descriptors and multiscale distance matrix for leaf recognition." In: Proceedings of International Symposium on ELMAR, pp. 23-26, Zadar, Croatia, Sept 2013.

[6] O. Mzoughi, I. Yahiaoui, N. Boujemaa, and E. Zagrouba, "Semanticbased automatic structuring of leaf images for advanced plant species identification," Multimedia Tools and Applications, vol. 75, no. 3, pp. 1615-1646, 2016.

[7] Aakif , M.F. Khan ," Automatic classification of plants based on their leaves", Biosyst. Eng. 139 (2015) 66-75 .

[8] Kadir, A., Nugroho, L.E., Susanto, A., Santosa, P.I., " Leaf classification using shape, color,and texture features." Int. J. Comput. Trends Technol. 1(3), 225-230 (2011).

[9] A. Olsen, S. Han , B. Calvert, P. Ridd, O. Kenny, "In situ leaf classification using histograms of oriented gradients", in: International Conference on Digital Image Computing, 2015, pp. 1-8 .

[10] Z. Tang, Y. Su, M.J. Er, F. Qi , L. Zhang, J. Zhou, "A local binary pattern based texture descriptors for classification of tea leaves", Neurocomputing 168 (2015) 1011-1023.

[11] G.L. Grinblat, L.C. Uzal , M.G. Larese , P.M. Granitto ," Deep learning for plant identification using vein morphological patterns", Comput. Electron. Agric. 127 (2016) 418-424 .

[12] Mairal, J., Bach, F., Ponce, J., Sapiro, G.: "Online learning for matrix factorization and sparse coding”. J. Mach. Learn. Res 11, 19-60 (2010).

[13] Aharon, M., Elad, M., Bruckstein, A.M.," The K-SVD: an algorithm for designing of overcomplete dictionaries for sparse representation”. IEEE Trans. Sig. Process. 54(11), 4311-4322 (2006).

[14] Beleites, C. and Neugebauer, U. and Bocklitz, T. and Krafft, C. and Popp, J.: "Sample size planning for classification models". Anal ChimActa, 2013, 760,2533.DOI:10.1016/j.aca.2012.11.007accepted manuscript on arXiv: 1211.1323.

[15] R. Rubinstein, M. Zibulevsky, and M. Elad, "Learning Sparse Dictionaries for Sparse Signal Approximation", Technical Report - CS, Technion, June 2009.

[16] Rodgers, J. L.; Nicewander, W. A. (1988). "Thirteen ways to look at the correlation coefficient". The American Statistician. 42 (1): 59-66. doi:10.1080/00031305.1988.10475524. JSTOR 2685263.

[17] The leaf image dataset available from http://sourceforge.net/projects/flavia/ files/.

[18] J. Sulam, B. Ophir, M. Zibulevsky and M. Elad, "Trainlets: Dictionary Learning in High Dimensions", IEEE Transactions on Signal Processing, Volume: 64, Issue: 12, June15, 2016. 